\title{
An equation to predict maximum acceptable loads for repetitive tasks based on duty cycle: evaluation with lifting and lowering tasks
}

\author{
Jim R. Potvin ${ }^{\mathrm{a}^{*}}$ \\ ${ }^{a}$ Department of Kinesiology, McMaster University, 1280 Main Street West, Hamilton, Ontario, \\ Canada, L8S $4 K 1$
}

\begin{abstract}
Recently, an equation was developed to predict maximal acceptable effort (MAE) for repetitive tasks based on the product of task frequency and effort duration (ie. duty cycle). This equation has been shown to closely match data from psychophysical studies of the upper extremities. In the current paper, the applicability of this equation was tested on lifting and lowering data from Snook and Ciriello (1991) and was found to fit closely, even at very low duty cycles.
\end{abstract}

Keywords: psychophysics, strength, maximum acceptable effort, ergonomic limits

\section{Introduction}

Physical ergonomists must determine acceptable demands for a variety of tasks. In rare cases, the tasks are performed very infrequently such that they are likely limited by maximum strength. However, in most cases, the tasks are repetitive and submaximal forces should be recommended. While a large biomechanical database exists in the literature to determine maximum strength, far fewer repetitive tasks have psychophysical and physiological data available to predict acceptable submaximal, repeated loads.

Recently, an equation has been developed by Potvin (2011) to predict maximal acceptable effort (MAE) based on the product of task frequency and effort duration (ie. duty cycle or DC). This equation was based on 69 values from 7 different psychophysical studies of various upper extremity tasks. There were seven strict criteria for inclusion in the equation development. The equation (Eqs. 1) was as follows:

$$
M A E=1-\left[D C-\frac{1}{28,800}\right]^{0.24}
$$

Where:

- MAE: Average maximum acceptable effort, where 1.0 represents $100 \%$ MVE.

- DC: Duty cycle where 1.0 represents $100 \%$ of the cycle

- 28,880 is the number of seconds in 8 hours. Its inverse is 0.0000347 and, when subtracted from $\mathrm{DC}$, results in $\mathrm{MAE}=1.0(100 \%)$ for all $\mathrm{DC} \leq 1$ s/workday.

With a best-fit exponent of 0.24 , the equation predicted MAE very well $\left(\mathrm{r}^{2}=0.87\right.$, RMS difference $=$ $7.2 \%$ of the maximum strength). At higher DC values, the equation also demonstrated good agreement with the published physiological data. The purpose of this paper was to evaluate the applicability of Eqs. 1 for determining maximum acceptable loads (MAL) for other tasks, specifically the lifting and lowering conditions presented by Snook and Ciriello (1991).

\footnotetext{
* Corresponding author. E-mail: potvinj@mcmaster.ca
} 


\section{Methods}

\subsection{Definitions}

Maximum acceptable effort (MAE) refers to an average maximum acceptable force or torque, normalized as a percentage of the average maximum force or torque (ie. strength) from a single maximum voluntary effort (MVE) for a specific task. For the purposes of this paper, this was assumed to be the maximum lift or lower load acceptable (MAWL) for one effort per, which was assumed to be ergonomically acceptable. Frequency is the number of discrete efforts per minute (ie. lifts/lowers). Duty cycle (DC) is the percentage of time an individual is performing efforts. For example, if a worker performs 4 lifts within each 62 second cycle, and each lift is 0.95 seconds in duration, then the duty cycle is (4 efforts/cycle $\times 0.95 \mathrm{~s} /$ effort $) / 62 \mathrm{~s}$ per cycle, or $\mathrm{DC}=$ 0.061 (6.1\% of each cycle). In the equation, it would be predicted that someone could lift/lower $48.8 \%$ of the 1 /day MAWL.

\subsection{Lifting and lowering psychophysical studies}

Snook and Ciriello (1991) summarized the results from eleven Liberty Mutual psychophysical studies of manual materials handling tasks including: lifting, lowering, pushing, pulling and carrying. Each study involved weeks of training before the completion of test adjustment sessions of at least four hours in duration. For a wide variety of task conditions, subjects determined maximum acceptable weights or forces. These are some of the only psychophysical studies to assess tasks with frequencies less than one per minute and, thus, challenged Eqs. 1 at very low DCs not used in its development. For the purposes of the current analysis, I used only the lifting and lowering data, as it was more difficult to estimate effort duration for the pushing, pulling and carrying tasks. For both lifting and lowering, I used all 216 combinations of three lifting/lower ranges (floor-knuckle, knuckleshoulder, shoulder-arm reach), three box widths (34, $49,75 \mathrm{~cm})$, three vertical displacements $(25,51,76$ $\mathrm{cm})$ and eight frequencies ( $\mathrm{f}=0.0021,0.033,0.20$, $0.50,1.00,4.39,6.67$ and $12.00 / \mathrm{min})$.

I only used the 50th percentile (mean) values and normalized all MAWL values with the corresponding MAWL at the lowest frequency (1 lift or lower/day). I performed a sensitivity analysis and concluded that the mean normalized values were similar for males and females, across box widths and across vertical displacements. Thus, I pooled values across these conditions to calculate six averages at each frequency (both lifting and lowering in the floor-to-knuckle, knuckle-to-shoulder and shoulder-to-reach ranges). Based on assumptions of a vertical velocity of 1.16 $\mathrm{m} / \mathrm{s}$ (Schipplein et al. 1990) and an average vertical displacement of $0.51 \mathrm{~m}$ with some added time for the initial displacement, I assumed the average lifting and lowering duration to be $0.50 \mathrm{~s}$ when calculating the duty cycles for each condition.

\subsection{Analysis}

There were a total of 42 lifting/lowering MAWL values evaluated from Snook \& Ciriello (1991)(Fig. 1a). For each value, I calculated the corresponding DC by multiplying the frequency by the estimated effort duration, and the MAE by dividing the task MAWL by the MAWL for a frequency of $1 /$ day.

The DC values were then input into the equation and compared to the calculated MAE value and evaluated with an RMS difference and $\mathrm{r}^{2}$ correlation.

\section{Results}

As with the upper extremity data, there was a strong, non-linear, negative relationship between DC and average MAE (Fig 1b). The equation had an RMS difference with the actual MAE of $5.0 \%$ and $\mathrm{r}^{2}$ $=0.812$ for the lifting/lowering data (compared to $7.2 \%$ and 0.874 for the upper extremity values). Overall, the RMS difference was 6.7\% MVE and the $\mathrm{r}^{2}=0.914$ across the 111 combined upper extremity and manual materials handling tasks.

Note that the predicted MAE curve decreases very rapidly in the very low range of DCs, but this follows the lifting/lowering data quite closely (Figure 1b, inset). While the predicted MAE is assumed to be $100 \%$ of maximum at $\mathrm{DC}=0.0000347$ (1s per 8 hours), it decreases substantially to $66.9 \%$ MVE at a $\mathrm{DC}=0.01(0.6 \mathrm{~s} / \mathrm{min})$. This corresponds very well to the Snook and Ciriello (1991) data where increases in DC to $0.00025,0.00167,0.00417$ and $0.00833(7,48$, 120 and 240 seconds per 8 hour work day) resulted in rapid decreases in the average MAWL to $80.0 \%$, $75.6 \%, 71.2 \%$ and $64.4 \%$, respectively, of the one per day maximum MAWL. 

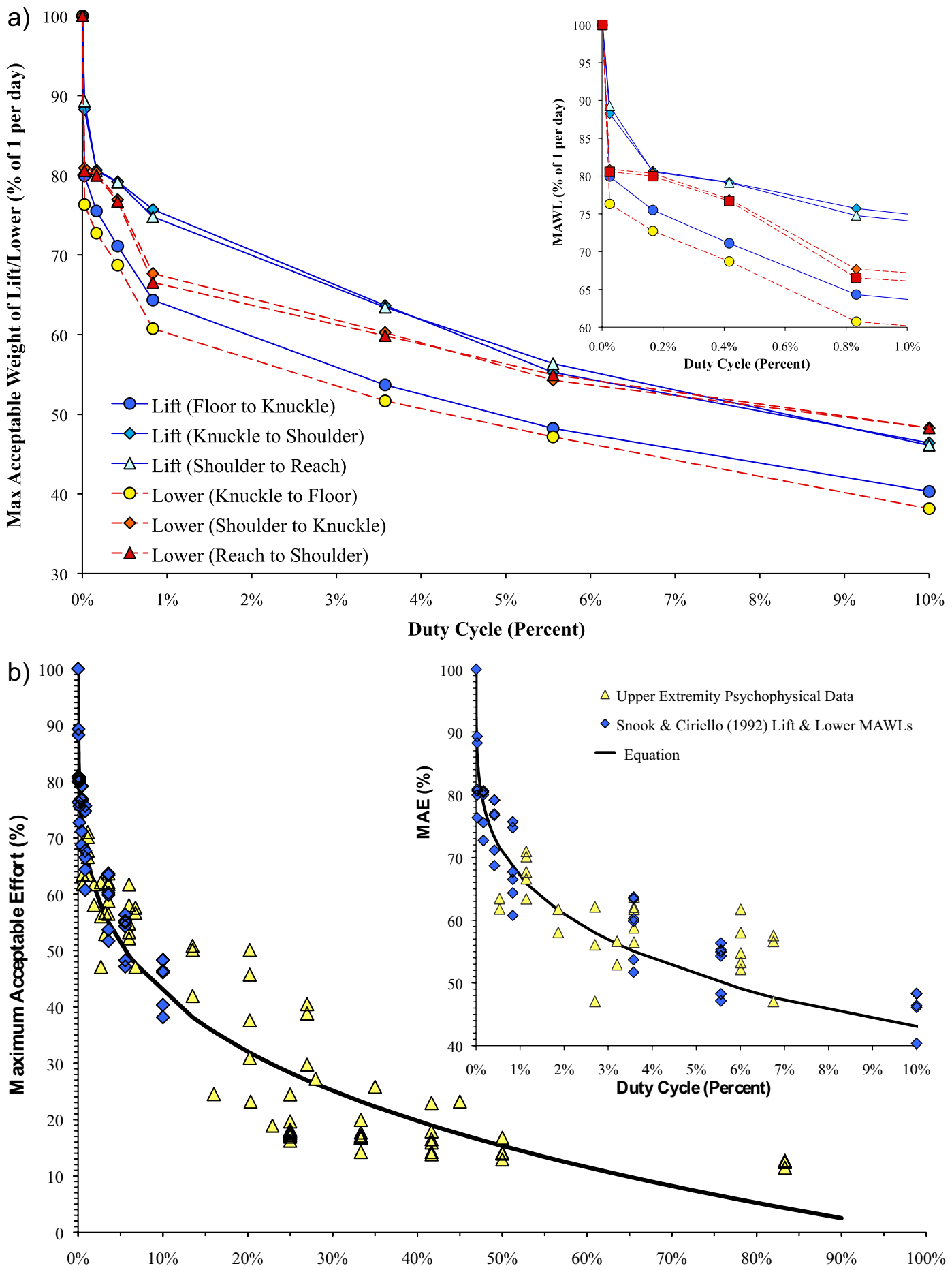

Duty Cycle (Percent)

Fig. 1: a) MAWL of lift and lower (as a percent of the 1/day MAWL) versus duty cycle. Data are shown from Snook and Ciriello (1991). Inset is a zoomed representation of the curve at the lowest duty cycles. b) MAE (as a percent of MVE) versus duty cycle as a percent of an 8-hour workday. All upper extremity $(n=69)$ and lift/lower psychophysical data $(n=42)$ are shown along with the curve from the proposed equation. Inset is a zoomed representation of the curve at the very low duty cycles. 


\section{Discussion}

This paper demonstrates the similarities in the general trend for decreases in MAE with increases in DC, for both manual materials handing and upper extremity tasks.

It is very interesting to see such similar relationships for whole body tasks (ie. lifting and lowering), and tasks involving only the upper extremities (ie. like wrist flexion, extension and ulnar deviation, pronation and supination, pushes with the finger tip and a pulp pinch and hose insertions). This bodes well for the general application of such an equation, at least for efforts of 10 seconds or less, as $10 \mathrm{~s}$ was the longest effort used for the development of Eqs. 1.

The equation fit well, even at the very lowest DCs $(<0.32$ seconds per minute) that were not even used in its development. In fact, the optimal exponent remains 0.24 if you only use the upper extremity data, as in Potvin (2011), or it is combined with the lifting and lowering data.
There are some limitations and constraints to the use of Eqs. 1. First, there were assumptions made about the duration of the lifting and lowering tasks in Snook and Ciriello (1991), however they were made based on empirical data and it is not expected that the actual values would have deviated substantially from the assumed values. Also, the equation should only be used for tasks that are repetitive without being combined with other tasks (eg. lifting plus carrying loads). Finally, it was assumed that maximum voluntary efforts would be acceptable, and meet ergonomic guidelines, only if performed very infrequently (ie. for one second or less each work day.

\section{References}

[1] J.R. Potvin, Predicting maximum acceptable efforts for repetitive tasks: an equation based on duty cycle, Human Factors (2011, in press)

[2] S.H. Snook, V.M. Ciriello, The design of manual handling tasks: Revised tables of maximum acceptable weights and forces. Ergonomics, 34(9), 1991, 1197-1213. 